\title{
Correlation Between Walking Ability and Monthly Care Costs in Elderly Patients After Surgical Treatments for Hip Fractures
}

\author{
Koki Abe, $\mathrm{MD}^{1,2}$, Kazuhide Inage, $\mathrm{MD}, \mathrm{PhD}^{1}$, Keishi Yamashita, $\mathrm{MD}^{2}$, Masaomi Yamashita, $\mathrm{MD}, \mathrm{PhD}^{2}$, \\ Akiyoshi Yamamaoka, $\mathrm{MD}^{2}$, Masaki Norimoto, $\mathrm{MD}^{1,2}$, Yoshinori Nakata, $\mathrm{MD}, \mathrm{PhD}^{3}$, \\ Takeshi Mitsuka, $\mathrm{MD}, \mathrm{PhD}^{4}$, Kaoru Suseki, $\mathrm{MD}, \mathrm{PhD}^{5}$, Sumihisa Orita, $\mathrm{MD}, \mathrm{PhD}^{1}$, \\ Kazuki Fujimoto, $\mathrm{MD}, \mathrm{PhD}^{1}$, Yasuhiro Shiga, $\mathrm{MD}, \mathrm{PhD}^{1}$, Hirohito Kanamoto, $\mathrm{MD}, \mathrm{PhD}^{1}$, \\ Masahiro Inoue, $\mathrm{MD}^{1}$, Hideyuki Kinoshita, $\mathrm{MD}^{1}$, Tomotaka Umimura, $\mathrm{MD}^{1}$, Yawara Eguchi, $\mathrm{MD}, \mathrm{PhD}^{6}$, \\ Takeo Furuya, MD, $\mathrm{PhD}^{1}$, Kazuhisa Takahashi, $\mathrm{MD}$, $\mathrm{PhD}^{1}$, Seiji Ohtori, MD, $\mathrm{PhD}^{1}$
}

${ }^{1}$ Department of Orthopedic Surgery, Graduate School of Medicine, Chiba University, Chiba City, Chiba; ${ }^{2}$ Department of Orthopedic Surgery, JCHO Funabashi Central Hospital, Funabashi City, Chiba; ${ }^{3}$ Nakata Orthopedics Clinic, Funabashi City, Chiba; ${ }^{4}$ Mitsuka Rheumatism Clinic, Narashino City, Chiba; ${ }^{5}$ Takanecho Orthopedics Clinic, Chiba City, Chiba;

${ }^{6}$ National Hospital Organization Shimoshizu Hospital, Yotsukaido City, Chiba, Japan

\begin{abstract}
Objective To validate the relationship between residual walking ability and monthly care cost as well as long-term care insurance (LTCI) certification level in elderly patients after surgical treatment for hip fractures in Japan.

Methods Elderly patients aged $>75$ years who underwent surgical treatment for hip fractures in our hospital were included. The preand post-surgical (6-month) walking ability and LTCI certification and the presence or absence of dementia was determined from medical records and questionnaires. Walking ability was classified into 6 levels used in our daily medical practice. Based on these data, we correlated the relationship between walking ability and the LTCI certification level. Further, based on the official statistics pertaining to the average monthly costs per person at each LTCI certification level, we evaluated the relationship between walking ability and monthly care cost.

Results A total of 105 cases (mean age, 80.2 years; 16 men; 39 patients with dementia) were included. The correlation between walking ability and average monthly cost per person as well as LTCI certification level at 6 months postoperatively ( $\mathrm{r}=0.58)$ was demonstrated. The correlation was found in both groups with and without dementia.

Conclusion The ability to walk reduced the cost of care in elderly patients who experienced hip fracture, regardless of the presence of dementia.
\end{abstract}

Keywords Hip fractures, Dementia, Long-term care, Mobility limitation, Cost-benefit analysis

Received April 28, 2017; Accepted September 19, 2017

Corresponding author: Koki Abe

Department of Orthopaedic Surgery, Graduate School of Medicine, Chiba University, 1-8-1 Inohana, Chuo-ku, Chiba City, Chiba 260-8670, Japan. Tel: +81-43-226-2117, Fax: +81-43-226-2116, E-mail: abeabeabe04@yahoo.co.jp

ORCID: Koki Abe (http://orcid.org/0000-0003-2259-3989); Kazuhide Inage (http://orcid.org/0000-0003-0540-4613); Keishi Yamashita (http://orcid. org/0000-0001-6988-0024); Masaomi Yamashita (http://orcid.org/0000-0002-6825-7182); Akiyoshi Yamaoka (http://orcid.org/0000-0002-0929-6747); Masaki Norimoto (http://orcid.org/0000-0003-4827-4062); Yoshinori Nakata (http://orcid.org/0000-0002-3912-6044); Takeshi Mitsuka (http://orcid. org/0000-0002-5566-2736); Kaoru Suseki (http://orcid.org/0000-0001-7471-9841); Sumihisa Orita (http://orcid.org/0000-0003-2676-5469); Kazuki Fujimoto (http://orcid.org/0000-0002-3118-9366); Yasuhiro Shiga (http://orcid.org/0000-0003-4887-0008); Hirohito Kanamoto (http://orcid.org/00000003-1194-3111); Masahiro Inoue (http://orcid.org/0000-0002-9518-9189); Hideyuki Kinoshita (http://orcid.org/0000-0002-0515-088X); Tomotaka Umimura (http://orcid.org/0000-0003- 4717-2849); Yawara Eguchi (http://orcid.org/0000-0003-2247-5376); Takeo Furuya (http://orcid.org/00000002-5378-940X); Kazuhisa Takahashi (http://orcid.org/0000-0002-1707-6656); Seiji Ohtori (http://orcid.org/0000-0001-5738-6184).

(c) This is an open-access article distributed under the terms of the Creative Commons Attribution Non-Commercial License (http://creativecommons.org/ licenses/by-nc/4.0) which permits unrestricted noncommercial use, distribution, and reproduction in any medium, provided the original work is properly cited. Copyright ( 2018 by Korean Academy of Rehabilitation Medicine 


\section{INTRODUCTION}

The general budget of Japan for the 2012 fiscal year was approximately $¥ 90$ trillion. The annual sum allotted to the long-term care insurance (LTCI) system established in 2000 steadily increased to $¥ 8.9$ trillion in the 2012 fiscal year, and is difficult to sustain [1]. The Nursing Care Needs Certification board, which consists of physicians, nurses, and other experts, determines the certification levels of LTCI, to comply with the needs of elderly persons aspiring to receive benefits [2]. The certification levels are stamped 'support levels 1 and 2' and 'care levels 1 to 5'. Support levels are milder than care levels and a lower number indicates milder levels. The higher the certified care level, the higher the average cost of care per patient in Japan's LTCI system [3]. Therefore, lowering the long-term care needs is an urgent goal to reduce LTCI costs.

Orthopedic surgeons and therapists endeavor to recover and maintain their patients' ability to walk. Nearly $94 \%$ of all elderly patients with hip fractures in Japan are treated surgically despite the high costs associated with surgery and the risks of co-morbidity $[4,5]$. In contrast, the LTCI certification level is not based on walking ability but on the number of labor hours required by the patient. For example, patients who require a wheelchair to move but can independently perform daily activities are regarded as mild cases. Conversely, patients with dementia who walk freely following surgical treatment are considered high-ticket recipients in the LTCI system. Therefore, a contrasting perspective of the LTCI system and the orthopedic surgeons exists with respect to patient needs. The former focuses on the estimated hours of care required by the patient, whereas the latter considers the recovery of musculoskeletal function, i.e., walking ability, as the main goal [2]. It is annoying that the LTCI system disregards walking ability compared with coexisting dementia during certification. Thus, we considered whether surgical orthopedic intervention reduces LTCI costs. Kim et al. [6] reported that motor impairment correlated with the service time provided to assist with activities of daily living (ADLs) in the LTCI system of Korea. However, few reports have investigated the correlation between walking ability and LTCI certification level in Japan's clinical setting. Thus, we investigated the relationship between residual walking ability and monthly costs per person at each LTCI certification level among patients with and without dementia in our medical district in Japan.

Another concern is that interventions in injured patients with dementia might not play a useful role in gait recovery because, dementia per se leads to ADL impairment $[7,8]$. Therefore, we compared the altered levels of walking ability from pre-injury stage to 6 months postinjury between patients with and without dementia.

\section{MATERIALS AND METHODS}

The present study protocol was reviewed and approved by the Ethics Committee of JCHO Funabashi Central Hospital (No. H28-7). Written informed consent was obtained from each patient. Elderly patients ( $>75$ years) who underwent surgery for hip fractures in our hospital from October 2011 to September 2013 were included. Pre-injury and postoperative (6-month) data related to walking ability and LTCI certification level were collected. Walking ability was classified into 6 levels used in daily medical practice: level 1, can walk freely (Free); level 2, can walk with a cane (Cane); level 3, can walk with a walker (Walker); level 4, can walk along a grab bar (Bar); level 5, can ambulate using a wheelchair (Wheel); and level 6, unable to walk and/or bedridden (Bed). Further, all patients were divided into the 8 LTCI certification levels formulated by the Ministry of Health, Labour and Welfare of Japan (in ascending order starting from mild cases): NA, not applicable; SL1 and SL2, support levels 1 and 2, respectively; CL1, CL2, CL3, CL4, and CL5, care levels 1 to 5, respectively. Data were gathered from the hospital medical records and the follow-up clinical questionnaires. Patients who failed to return for follow-up were contacted by phone or via their nursing care managers to the best possible extent. Injured elderly patients who were characterized with disquiet mood, turbulent behavior, memory loss, difficulties with thinking, or history of dementia were defined as patients with dementia. Furthermore, surgical expenses including material costs were surveyed from the account ledgers of the hospital.

Based on these data, we investigated the changes in levels of walking ability from pre-injury to 6 months after surgery, correlation between levels of walking ability and LTCI certification at 6 months postoperatively, and correlation between levels of walking ability and the average monthly cost of care per person at each LTCI certification 
level. Additionally, we compared these points between patients with dementia (dementia group) and without dementia (non-dementia group). Mann-Whitney U test, Wilcoxon signed-rank test, and Spearman correlation coefficient by rank test were used for statistical analysis ( $\mathrm{p}<0.05$ indicated significance).

Regarding insurance costs LTCI supplied to in-home care cases, the Ministry of Health, Labour and Welfare reported that the average monthly cost per person at each LTCI certification level was as follows: SL1 (¥22,842), SL2 $(¥ 41,802)$, CL1 (¥73,628), CL2 (¥102,118), CL3 (¥154,020), CL4 (¥187,684), CL5 (¥230,062) [3]. Based on these values, we evaluated the correlation between care costs and levels of walking ability following surgery for hip fractures in our elderly cohort.

\section{RESULTS}

A total of 138 patients aged $>75$ years experienced hip fractures and underwent surgical treatment in our hospital during the selected 2-year period. Eight patients who died during the study period-1 died on postoperative day 6 due to sepsis and 7 who died of internal disease ( 3 cases of pneumonitis and 1 case each of bladder cancer, thrombosis of the superior mesenteric artery, hematological disease, and decrepitude) after dischargewere excluded. Another 25 cases were lost to follow-up, and were also excluded. Finally, we obtained valid data pertaining to 105 cases. In this cohort, 48 patients with femoral neck fractures were included, including 11 who underwent internal fixation and 37 who were treated with hemiarthroplasty (Table 1). Fifty-five patients sustained intertrochanteric fractures, and 52 of them underwent internal fixation and 3 were treated with hemiarthroplasty. Two patients with sub-trochanteric fractures underwent internal fixation. The mean age was $\mathbf{8 0 . 2}$ years (range, 75-102 years), and 16 patients were male. Thirty-nine patients experienced dementia as a complication (dementia group) and 66 did not manifest dementia (non-dementia group). All cases underwent physical therapy starting on admission or on the next day. The wait time from injury to surgery was $4.35 \pm 2.22$ days, which was not significantly different between the dementia and non-dementia groups (3.92 \pm 2.25 and $4.56 \pm 2.13$ days, respectively). The mean operating cost (including material costs) was $¥ 696,000$.

\section{Walking ability}

In the entire cohort, the walking ability of patients before injury was as follows: level 1 (Free), 65; level 2 (Cane), 14; level 3 (Walker), 8; level 4 (Bar), 14; level 5 (Wheelchair), 2; level 6 (Bed), 2 patients. Six months postoperatively, the patients' walking ability was as follows: $31,26,10,17,15$, and 6, respectively. As a whole, walking ability at 6 months postoperatively was significantly worse compared with pre-injury level $(1.86 \pm 1.30$ and $2.78 \pm 1.62$, respectively; $\mathrm{p}<0.05)$. The average walking ability before injury and at 6 months postoperatively was

Table 1. Patients' demographics ( $\mathrm{n}=105)$

\begin{tabular}{|c|c|c|c|}
\hline Characteristic & Dementia cases $(n=39)$ & Non-dementia cases $(n=66)$ & p-value \\
\hline Age (yr) & $87.7 \pm 5.34$ & $83.1 \pm 5.77$ & $<0.01^{*}$ \\
\hline Sex (male:female) & $6: 33$ & $15: 51$ & NS \\
\hline \multicolumn{4}{|l|}{ Diagnosis } \\
\hline Femoral neck fracture & $15(38.5)$ & $33(50.0)$ & \\
\hline Inter-trochanteric fracture & $23(58.9)$ & $32(48.5)$ & \\
\hline Sub-trochanteric fracture & $1(2.6)$ & $1(1.5)$ & \\
\hline \multicolumn{4}{|l|}{ Treatment } \\
\hline ORIF & $28(71.8)$ & $37(56.1)$ & \\
\hline ORIF (femoral neck fracture) & $6(15.4)$ & $5(7.6)$ & NS \\
\hline Hemiarthroplasty & $11(28.2)$ & $29(43.9)$ & \\
\hline Waiting time (day) & $3.92 \pm 2.26$ & $4.56 \pm 2.13$ & NS \\
\hline
\end{tabular}

Values are presented as mean \pm standard deviation or number (\%).

NS, no significant difference; ORIF, open reduction internal fixation.

${ }^{*} \mathrm{p}<0.05$ was considered statistically significant. 
$2.46 \pm 1.54$ and $3.49 \pm 1.62$, respectively, in the dementia group and $1.50 \pm 1.00$ and $2.36 \pm 1.49$, respectively, in the non-dementia group. The walking ability (pre-injury and 6 months post-injury) of patients with dementia was significantly lower than in patients without dementia $(\mathrm{p}<0.05)$. Although both dementia and non-dementia groups showed a significant decrease in walking ability during 6 months (decreased by $1.03 \pm 1.25$ and $0.86 \pm 1.39$ levels, respectively, $\mathrm{p}<0.05)$, the degree of change was not significant between the two groups (Fig. 1).

\section{LTCI certification level at 6 months postoperatively}

The LTCI certification levels at 6 months postoperatively were as follows: NA, 19; SL1, 8; SL2, 8; CL1, 15; CL2, 20; CL3, 19; CL4, 11; CL5, 5, with SL2, 2; CL1, 3; CL2, 9; CL3, 16; CL4, 4; CL5, 5; SL1, 0; NA, 0 in the dementia group and NA, 19; SL1, 8; SL2, 6; CL1, 12; CL2, 11; CL3, 3; CL4, 7; CL5, 0 in the non-dementia group. The LTCI certification levels of patients in the dementia group were significantly higher than those of patients in the non-dementia group $(\mathrm{p}<0.05)$.

\section{Relationship between walking ability and LTCI} certification level at 6 months postoperatively

A significant correlation existed between the levels of walking ability and certified levels in the LTCI system at 6 months postoperatively $(\mathrm{r}=0.58)$, with the regression curve at $\mathrm{Y}=0.75 \mathrm{X}+2.2$ (Fig. 2). Additionally, we detected a significant correlation between walking ability and LTCI certification levels ( $r=0.37$ and $r=0.61$, respectively) in both dementia and non-dementia groups.

\section{Relationship between walking ability and the average} monthly cost of care at 6 months postoperatively

Based on the official statistics pertaining to the average monthly cost at each LTCI certification level, a significant correlation existed between walking ability and the average monthly cost of care per patient at 6 months postoperatively $(\mathrm{r}=0.58)$, with the regression curve at $\mathrm{Y}=2.42 \times 10^{4} \mathrm{X}+2.70 \times 10^{4}$ (Fig. 3 ).

\section{DISCUSSION}

The results of this study showed that the walking ability of patients in the dementia group was inferior both before injury and at 6 months postoperatively and their LTCI levels at 6 months postoperatively were more severe compared with those of patients in the non-dementia group. These results reflect our experience in daily practice. However, no significant difference existed between

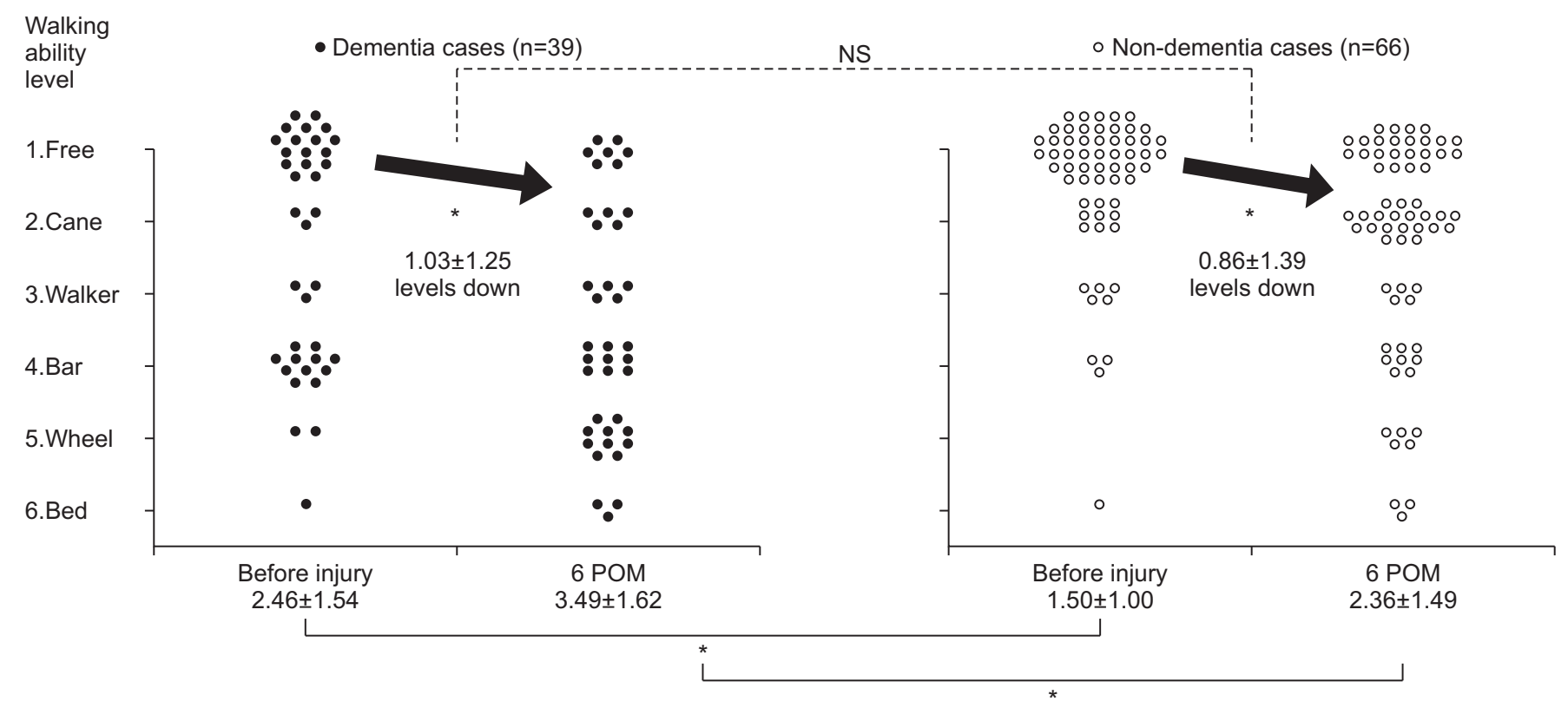

Fig. 1. Dot diagram showing the change in walking ability. Walking ability was classified as follows: ability to walk freely (Free), ability to walk with a cane (Cane), ability to walk with a walker (Walker), ability to walk along a bar (Bar), ability to ambulate using a wheelchair (Wheel), inability to walk and/or bedridden (Bed). NS, no significant difference; POM, months postoperatively. ${ }^{*} \mathrm{p}<0.05$. 


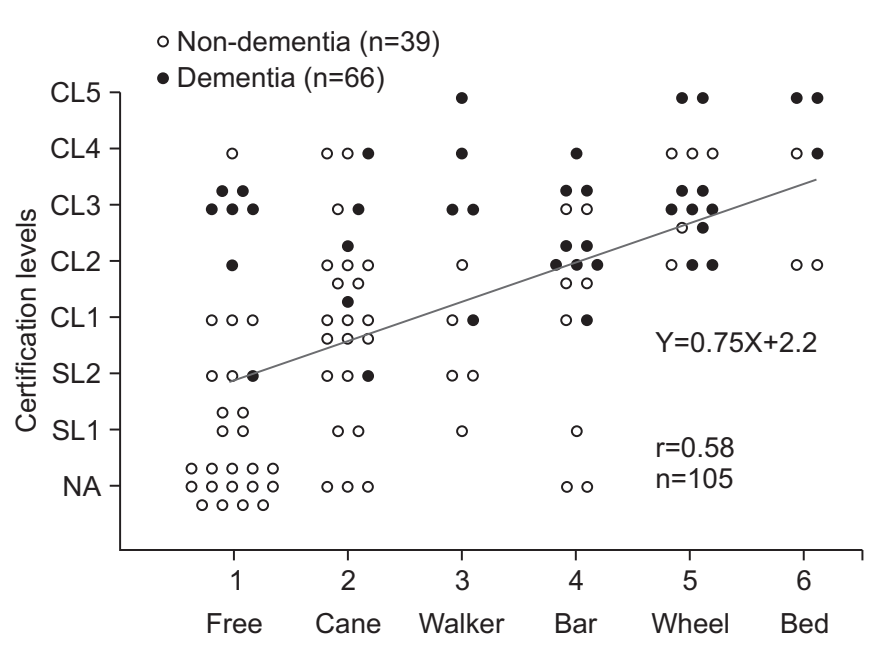

Fig. 2. Correlation between walking ability and insurance certification level. NA, not applicable; SL1 and SL2, support levels 1 and 2, respectively; CL1, CL2, CL3, CL4, and CL5, care levels 1 to 5, respectively.

these two groups about the variation of degree during 6 months, implying that surgical treatment of elderly patients with dementia resulted in similar effects compared with non-dementia cases. Notably, walking ability correlated with LTCI certification levels at 6 months after surgery regardless of the presence of dementia. Additionally, we found a significant correlation between walking ability and average monthly care costs at 6 months postoperatively (regression curve, $\mathrm{Y}=2.42 \times 10^{4} \mathrm{X}+2.70 \times 10^{4}$ ) (Fig. 3). A single-level improvement in walking ability indicated a $¥ 24,000$ reduction in LTCI expenses per patient per month. Considering that average surgical cost is $¥ 695,000$, if surgical treatment resulted in a level $1 \mathrm{im}$ provement in walking ability, the cost was offset within 29 months of surgery ( $¥ 24,000 \times 29$ months $=¥ 696,000$ ); however, the study lacked a control group. Previous studies reported that the survival rates of hip fracture at 2 and 3 years postoperatively in Japan were $67 \%-76 \%$ and $63 \%-69.3 \%$, respectively [9-11]. With favorable long-term survival prospects, the reduction in monthly care costs is very important.

This study has several limitations. First, we did not investigate the hospitalization period or the presence of complications aside from dementia. Second, despite the uncommon use of the 6-level classification of walking ability, we used it in the present study to correlate the degree of recovery of motor function with LTCI certification. However, we believe that this study provides valuable in-

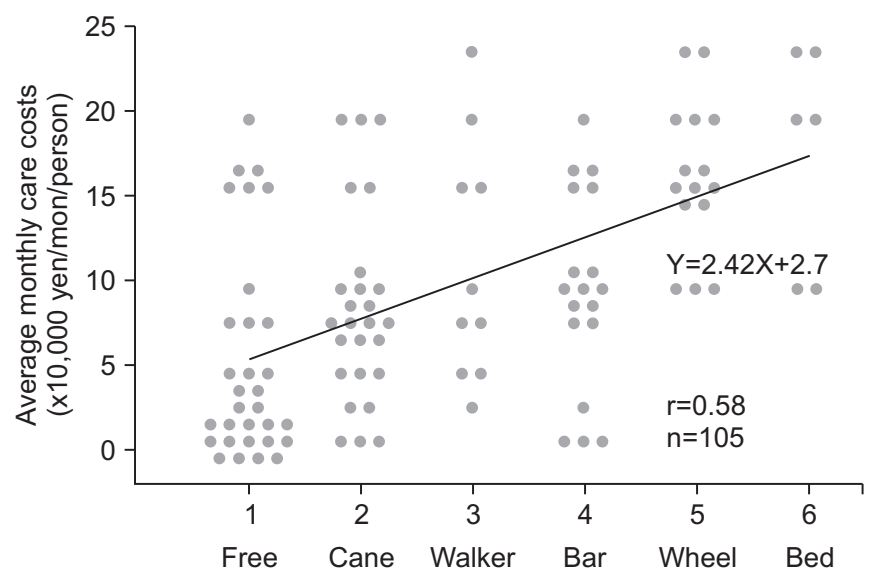

Fig. 3. Correlation between walking ability and average monthly care costs. The black straight line represents the regression curve.

formation correlating walking ability with long-term care costs in elderly patients following surgical treatment for hip fractures. Finally, the present study did not show the efficacy of surgical treatment. However, the study confirmed that the inherent ability to walk reduced the cost of care in elderly patients who experienced hip fracture, regardless of the presence of dementia. A comparative cost-benefit analysis of surgical and conservative treatments for elderly patients with hip fractures requires a larger-scale comprehensive study based on a common classification system that includes admission fee and survival rates after injury as well as residual walking ability, certified level of LTCI, and surgical costs as survey items.

\section{CONFLICT OF INTEREST}

No potential conflict of interest relevant to this article was reported.

\section{ACKNOWLEDGMENTS}

We thank Kikuo Takahashi, the assistant director of hospital, clerical worker Naomi Nozaki, and assistant head of home-visit nursing Masako Kawada for their expert advice and support, as well as JCHO Funabashi Central Hospital.

\section{REFERENCES}

1. Yamauchi Y. Decomposing cost efficiency in regional 
long-term care provision in Japan. Glob J Health Sci 2015;8:89-100.

2. Tsutsui T, Muramatsu N. Care-needs certification in the long-term care insurance system of Japan. J Am Geriatr Soc 2005;53:522-7.

3. Health, Labour and Welfare Statistics Association. Survey of long-term care benefit expenditures, FY2013, summary of annual report. J Health Welfare Stat 2014;61:51-7.

4. Hagino H, Sakamoto K, Harada A, Nakamura T, Mutoh Y, Mori S, et al. Nationwide one-decade survey of hip fractures in Japan. J Orthop Sci 2010;15:737-45.

5. Takamine B, Morii T, Watanabe H, Tajima T, Satomi K. Short-term postoperative mortality events in patients over 80 years of age with hip fracture: analysis at a single institution with limited medical resources. J Orthop Sci 2010;15:437-42.

6. Kim $\mathrm{YH}$, Kwon $\mathrm{CH}$, Shin HI. Validity of motor impairment scale in long-term care insurance system of Korea. Ann Rehabil Med 2013;37:403-12.

7. Njegovan V, Hing MM, Mitchell SL, Molnar FJ. The hierarchy of functional loss associated with cognitive decline in older persons. J Gerontol A Biol Sci Med Sci 2001;56:M638-43.

8. Iwasa H, Gondo Y, Yoshida Y, Kwon J, Inagaki H, Kawaai C, et al. Cognitive performance as a predictor of functional decline among the non-disabled elderly dwelling in a Japanese community: a 4-year population-based prospective cohort study. Arch Gerontol Geriatr 2008;47:139-49.

9. Miyanishi K, Jingushi S, Torisu T. Mortality after hip fracture in Japan: the role of nutritional status. J Orthop Surg (Hong Kong) 2010;18:265-70.

10. Takayama S, Iki M, Kusaka Y, Takagi H, Tamaki S. Rate of mortality with hip fracture and its prognostic factors in an elderly Japanese population. Environ Health Prev Med 2001;5:160-6.

11. Tsuboi M, Hasegawa Y, Suzuki S, Wingstrand H, Thorngren KG. Mortality and mobility after hip fracture in Japan: a ten-year follow-up. J Bone Joint Surg Br 2007;89:461-6. 